\title{
Impact of Fiber Nonlinearity on the Performance of Mode Division Multiplexing Systems
}

\author{
Esraa K. Hamed ${ }^{1}$, Mohammed A. Munshid ${ }^{2}$, Jassim K. Hmood ${ }^{3}$ \\ Laser and Optoelectronic Engineering Department, University of Technology, Baghdad, Iraq \\ esrakahtanre@yahoo.com,dr.mohhamedwhab@gmail.com,jassim_711@yahoo.com
}

\begin{abstract}
-in this paper, an analytical model is developed to estimate a nonlinear phase noise (NPN) due to Kerr fiber nonlinearity and its interaction with amplifier noise in mode division multiplexing (MDM) systems. Our analysis uses generalized coupled multimode nonlinear Schrödinger equations (MM- NLSE) that describe the propagation of the mode superimposing in the optical fiber. The nonlinear phase noise versus channel power and transmission distance is evaluated by implementing our analytical model for LP01, LP11a, and LPIIb spatial modes. Each mode carries a 4-QAM signal at a symbol rate of $20 \mathrm{Gsymbol} / \mathrm{s}$. The results reveal that LP11 mode has a lower phase noise variance than LP01 mode over entire transmission distances. Finally, the error vector magnitude (EVM) versus channel power is quantified using an analytical model.
\end{abstract}

Index Terms- Few-mode fibers, nonlinear phase noise, mode division multiplexing system, optical fiber nonlinearity.

\section{INTRODUCTION}

In order to overcome the capacity limitation of single mode fiber (SMF) communication systems, spacedivision multiplexing (SDM) has been introduced as an overgrown solution [1,2]. SDM systems have been realized by using multicore fibers (MCFs) or few-mode fibers (FMFs). Many signals can be spatially sent over multiple cores of multicore fiber. On the other hand, mode-division multiplexing (MDM) system can support multiple transmission modes over single-core FMF[3].

Mode-division multiplexing over FMFs has large-scale attention over the past few years because it can be considered as the next generation solution to overcome the capacity crunch of SMF [4-6]. FMF support multiple spatial modes that allow to carrying several channels over orthogonal modes of the FMF $[7,8]$, leading to increasing the capacity of the communication system. In addition, multi-core fibers with few-mode cores (FM-MCFs) have been proposed for increasing the overall number of transmitted channels[7, 9].

Various research works have focused on demonstrating the feasibility of the MDM systems and compensating linear distortions of the optical signal using digital signal processing (DSP) [10, 11]. Nonlinear propagation effects essentially limit optical fiber communication systems and their recognition for multimode fiber (MMF) is still restricted in comparison to SMF [12]. In silica glass fibers, the nonlinear impairments such as self-phase modulation (SPM) and cross-phase modulation (XPM) are caused as a result of the third-order Kerr nonlinearities. Due to sharing many spatial modes the core of FMFs, nonlinear fiber impairments are highly increased because of the interaction between different modes. The study of spatial/temporal nonlinear effect in (MMFs) has been considered as an important field of research over the past few years [13].

Normally, to compensate the fiber losses, optical amplifiers are employed periodically. However, the optical amplifier naturally produces an amplified spontaneous (ASE) noise, which is appended to the optical signal. Furthermore, the ASE noise interacts with Kerr fiber nonlinearity during the 
amplification process, generating a random nonlinear distortion that accumulated along fiber link[14]. The number and gain of the amplifiers play a significant role in the amount of induced nonlinear phase noise [15]. The main reason for degrading the system performance is a nonlinear phase noise that reduces the quality of the signal and limits the transmission distance [16].

Up to now, most studies have focused on modeling and investigating the effect of fiber nonlinearity on MDM system performance for short distances or without considering the interaction of ASE noise with SPM or XPM phenomenon. In this paper, we develop an analytical model to estimate NPN that produced by fiber nonlinearity and its interaction with ASE noise in coherent two-mode MDM systems. The analytical model concentrates on the nonlinear phase noise in single polarization m-array quadrature amplitude modulation (m-QAM). Our analytical model is capable to evaluate the NPN variance due to variations in channel power, transmission distance, and spatial modes.

The rest of this paper is organized as follows: in Section II, a general analytical model that describes the nonlinear propagation of optical signals in MMFs is developed and the phase noise induced by nonlinear impairments is derived. The analytical results are demonstrated and discussed in Section III. Finally, conclusions are given in Section IV.

\section{ANALYTICAL MODELING OF MDM SYSTEM}

In this section, an analytical model is developed for estimating the NPN in the long haul MDM system. Generalized coupled multimode nonlinear Schrödinger equations (MM- NLSE) can describe the transmission of multi-mode over optical fiber as [12].

$$
\frac{\partial A_{p}}{\partial z}=i\left(\beta_{p 0}-\beta_{r}\right) A_{p-}\left(\beta_{1 p}-\frac{1}{v_{g r}}\right) \frac{\partial A_{p}}{\partial t}-i \frac{\beta_{2 p}}{2} \frac{\partial^{2} A_{p}}{\partial t^{2}}-\frac{\alpha}{2} A_{p}+i \sum_{l m n} f_{\mathrm{lmmp}} \gamma A_{m} A_{n} A_{l}^{*} e^{i \Delta \beta_{i m p} z}+i \sum_{m} q_{n m} A_{m}
$$

Where:

$A_{p}$ is an envelope of the optical signal.

$\mathrm{v}_{\mathrm{gr}}$ is group velocity.

$\beta_{\mathrm{r}}$ is reference propagation constant.

$\alpha$ is the attenuation coefficient.

$\gamma$ is a Nonlinear coefficient.

$\beta_{0 \mathrm{p}}$ is propagation constant of $\mathrm{p}^{\text {th }}$ mode.

$\beta_{1 \mathrm{p}}$ inverse of the group velocity of the $\mathrm{p}^{\text {th }}$ mode.

$\beta_{2 \mathrm{p}}$ is second-order dispersion of the $\mathrm{p}^{\text {th }}$ mode.

$\mathrm{q}_{\mathrm{mp}}$ is linear mode-coupling coefficient.

$\mathrm{f}_{\text {lmnp }}$ is nonlinear mode-coupling coefficient.

$\Delta \beta_{\text {lmnp }}$ is phase mismatch between spatial modes.

The nonlinear coupling coefficient can be defined by the overlap integrals in (2) as [12]:

$$
\boldsymbol{f}_{\text {lmnp }}=\frac{A_{\text {eff }}}{\left(I_{l} I_{m} I_{n} I_{p}\right)^{2}} \iint F_{l}^{*} F_{m} F_{n} F_{p}^{*}
$$

Where $\boldsymbol{I}_{\boldsymbol{m}}$ and $\boldsymbol{F}_{\boldsymbol{m}}$ are the intensity and transverse field distribution of $m^{\text {th }}$ mode, respectively? Last part of (1) shows the linear coupling between modes where it arises because of the refractive index disturbance. The refractive index FMF is depended on the intensity of the propagated optical beam, leading to induce interesting nonlinear effects such as self-phase modulation (SPM), cross-phase modulation (XPM) and four-wave mixing (FWM). The fifth term in (1) represents these nonlinear effects and it can be expressed as: 


$$
\frac{\partial A_{p}}{\partial z}=i \sum_{l m n}^{N} f_{l m n p} \gamma A_{n} A_{m} A_{l}^{*} e^{i \llbracket \beta_{l m m p} z}
$$

Where $N$ is the number of spatial modes. SPM can be defined from (3) by setting modes indices equal $(l=m=n=p)$ while by placing the mode indices as either $(m=l$ and $n=p)$ or $(m=p$ and $n=l)$, the term express XPM. Other combinations for modes indices induce FWM phenomena. It can be also observed that, from (3), the nonlinear term is summed over the three indices $l, m, n$, each accounting from 1 to $N$. The number of these nonlinear terms grows like $N^{3}[17,18]$. SPM, XPM, and FWM phenomenon have been reported to be significant in long-haul multi-channel systems such as WDM and OFDM transmission systems[16, 19]. Phase degradation due to nonlinear effects and its interaction with ASE has been widely studied in amplified coherent communication systems [15, 20,21]. It has been observed that random NPN is induced by interacting SPM, XPM, and FWM phenomena with ASE inside optical amplifiers.

Let us consider the optical field of $p^{\text {th }}$ mode is given by

$$
u_{p}(z, t)=\left[u_{p}(0, t)\right] \exp \left(i \phi_{p}(t)\right)+n(t)
$$

where $\mathrm{u}_{\mathrm{p}}(0, \mathrm{t})$ is the transmitted signal at $p^{\text {th }}$ mode, $\mathrm{u}_{\mathrm{p}}(\mathrm{z}, \mathrm{t})$ is the propagated signal at distance of $\mathrm{z}, \mathrm{n}(\mathrm{t})$ is optical amplifiers noise, and $\phi_{p}(\mathrm{t})$ is the phase distortion due to dispersion and nonlinear effects for $p^{\text {th }}$ mode and it can be written as:

$$
\phi_{p}(t)=\phi_{p S P M}+\phi_{p X P M}+\phi_{p S P M}^{n(t)}+\phi_{p X P M}^{n(t)}+\phi_{p D I S}
$$

It can be expressed the envelope of m-QAM transmitted signal that launched to fiber as:

$$
u_{p}(0, t)=\sqrt{\frac{P_{0}}{2}} A_{p}(t)
$$

where $P_{0}$ is the maximum mode power (assume all modes have the same power), $A_{P}$ is the complex amplitude depending on the m-QAM constellation.

In a long-haul communication system, optical amplifiers are employed for compensating optical fiber losses. Various optical amplifiers have been constructed with different characteristics. However, ASE noise that originated from optical amplifiers is a major source of noise and it accumulates over transmission distance [22]. Noise term can be described as a summation of channels noises. It was considered as an additive white Gaussian noise.

$$
n(t)=\sum_{p}^{N} n_{p}(t) \exp \left(i w_{p} t\right)
$$

In MDM systems, the SPM occurs when the propagated optical field of any mode modulates its phase. Accordingly, a nonlinear phase is produced during the propagating signal in FMF. XPM is a nonlinear phase shift induced by fields of other modes that have a different propagation path or a state of polarization. FWM occurs when photons from other modes are interacted, producing new photons with different frequencies and modes in which the net energy and momentum are preserved [18]. Indeed, fiber nonlinearity effects are a result of changing in refractive index. The amount of changing in refractive index is relative to the intensity of the propagated optical signal, in which the changing in refractive index is $2 n_{2}\left|u_{p}\right|^{2}$. Here $n_{2}$ is second-order refractive index.

By solving (1), the NPN due to both SPM and XPM for N spatial modes that propagate through FMF can be written as: 


$$
\begin{aligned}
& \phi_{p S P M}(z)=2 \gamma L_{p p p p}(z) f_{p p p p} \sum_{\mu=1}^{M}\left|u_{p}\right|^{2} \\
& \phi_{p X P M}(z)=4 \gamma \sum_{l=1}^{N} L_{l l p p} f_{l l p p} \sum_{\mu=1}^{M}\left|u_{l}\right|^{2}
\end{aligned}
$$

where $M$ represents spans number, and

$$
L_{\text {lmnp }}(z)=\frac{1-\exp \left(-\left(\alpha+i \Delta \beta_{l m n p}\right) z\right)}{\left(\alpha+i \Delta \beta_{l m n p}\right)}
$$

Phase mismatch parameter between spatial modes can be given by:

$$
\square \beta_{l m n p}=-\beta_{l}(w)-\beta_{p}(w)+\beta_{m}(w)+\beta_{n}(w)
$$

It was calculated by analyzing the wave-number of contributing waves using Taylor series close to a reference frequency, $w_{0}$, as [23]:

$$
\beta_{p}(w)=\beta_{0 p}+\beta_{1 p}\left(w-w_{0}\right)+\frac{\beta_{2 p}}{2}\left(w-w_{0}\right)^{2}+\frac{\beta_{3 p}}{6}\left(w-w_{0}\right)^{3}+\ldots .
$$

where $\beta_{k p}$ is the $k^{\text {th }}$ order expansion coefficient for $p^{\text {th }}$ mode. The first, second and third derivatives, $\beta_{1}$, $\beta_{2}$ and $\beta_{3}$ that evaluated at $w_{0}$, characterize the inverse of group velocity, second-order dispersion, and third-order dispersion, respectively. Nonlinear coupling coefficients of the form $f_{p p p p}$ can be considered as coupling coefficients for SPM and all nonlinear coupling coefficients of the form $f_{l p l p}$ and $f_{l l p p}$ can be considered as coupling coefficients for XPM which having the same value. Other modes combinations can be assumed as coupling coefficients for the FWM process.

After some mathematical processes, we can describe the random NPN that induced by interreacting ASE noise with SPM and XPM inside amplifiers by:

$$
\begin{aligned}
& \phi_{p S P M}^{n(t)}(z)=2 \gamma L_{p p p p} f_{p p p p} \sum_{\mu=1}^{M} \Re\left(u_{p} \sum_{\kappa}^{\mu} n_{p \kappa}^{*}(t)\right) \\
& \phi_{p X P M}^{n(t)}(z)=4 \gamma \sum_{\substack{l=1 \\
p \neq l}}^{N} L_{l l p p} f_{l l p p} \sum_{\mu=1}^{M} \Re\left(u_{l} \sum_{\kappa}^{\mu} n_{p \kappa}^{*}(t)\right)
\end{aligned}
$$

where $\phi_{p S P M}^{n(t)}$ and $\phi_{p X P M}^{n(t)}$ are the NPNs due to both SPM and XPM phenomena for $\mathrm{p}^{\text {th }}$ mode, respectively and $n_{p \kappa}^{*}(t)$ is complex conjugated of the amplifier noise at $p^{\text {th }}$ mode and $\mu^{\text {th }}$ span. Here $\mathfrak{R}(\mathrm{x})$ refers to the real value of $\mathrm{x}$. The phase noise induced by dispersion can be expressed as [16]:

$$
\phi_{p D I S}=-\left(\frac{\beta_{2 p}}{2} w_{p}^{2}\right) L
$$

\section{RESULTS AND DISCUSSION}

In this section, the impact of fiber nonlinearity on the 2-mode 4-QAM MDM system is studied. The influences of channel power and transmission distance on the NPN variation are demonstrated. Furthermore, the effect of launched power on EVM is demonstrated. To evaluate NPN, a multi-span MDM transmission system that supports LP01, LP11a, and LP11b spatial modes are considered. Each spatial mode carries a 4-QAM signal at a symbol rate of $20 \mathrm{Gsymbol} / \mathrm{s}$ and propagates over a gradedindex FMF. 
In our analysis, we consider all modes suffer from strong linear mode coupling. The FMF is designed with $\alpha=0.22 \mathrm{~dB} / \mathrm{km}$ (assumed to be the same for all modes) and the nonlinear coefficient is fixed at $1.77 \mathrm{~W}^{-1} \cdot \mathrm{km}^{-1}$. The values of chromatic dispersion of both LP01 and LP11 are set to 19.1 and 20.3 $\mathrm{ps} /(\mathrm{nm} . \mathrm{km})$, respectively. Relative inverse group velocity is set to $300 \mathrm{ps} / \mathrm{km}$ for LP01 and $0 \mathrm{ps} / \mathrm{km}$ for LP11 at $1550(\mu \mathrm{m})$. The transmission link is considered as a multi-span optical link. Each span includes FMF with a length of $50 \mathrm{~km}$, one Erbium-doped fiber amplifier (EDFA) and dispersion-compensating fiber (DCF). EDFA totally compensates the attenuation and its noise figure is fixed to $6 \mathrm{~dB}$ while the dispersion is fully compensated by DCF with a length of $5 \mathrm{~km}$.

\section{A. Phase noise versus mode power}

The flowchart is shown in Fig. 1 represents the main steps of estimation both SPM and XPM phase noise variances versus mode power variation. Figures 2(a) and 2(b) display the variances of NPN of SPM and XPM in presence ASE noise against the mode power for 4-QAM MDM system, respectively. The length of the fiber link is adjusted to $250 \mathrm{~km}$. We can observe that, at low mode power, the NPN variance is primarily induced by ASE noise since the mode power is low. With rising mode power, the NPNs by both SPM and XPM are exponentially increased since Kerr fiber nonlinearity is depended on the intensity of the signal. In contrast with LP01, LP11a and LP11b spatial modes are less affected by both SPM and XPM phenomena due to their larger effective areas and higher differential group delay. Furthermore, the interaction of XPM with optical amplifier noise has a higher contribution to NPN for 2-mode MDM system.

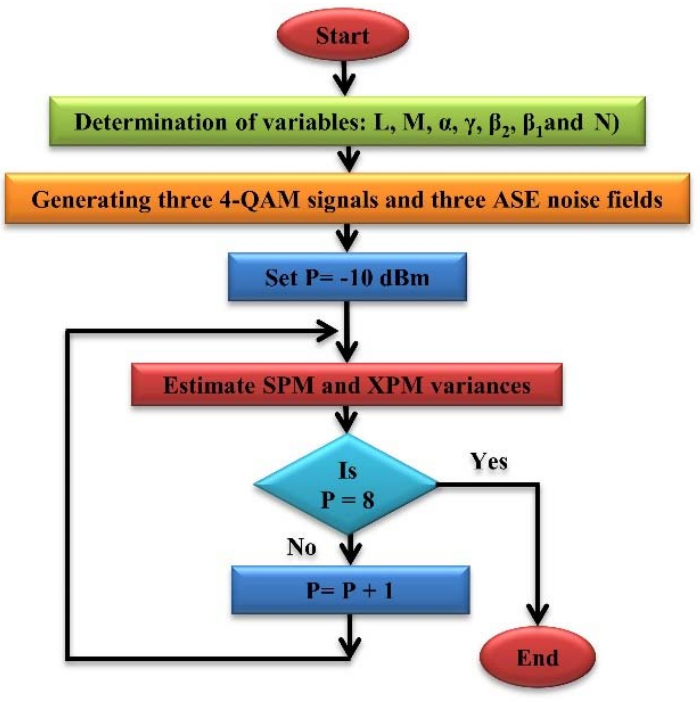

Fig.1. FlowChART OF ESTimation NPN VARIANCE.

As depicted in Fig. 2(c), total variance of NPNs initially decays as mode power is increased. Nevertheless, with increasing channel power over $-1 \mathrm{dBm}$ and $0 \mathrm{dBm}$ for LP01 and LP11, respectively; the variance of NPN starts to rise because the amount of NPN is depended on the intensity of transmitted optical signals. For 2-mode 4-QAM MDM system, minimum phase noise variances of $\left(7.82 \times 10^{-4} \mathrm{rad}^{2}\right)$ and $\left(6.4 \times 10^{-4} \mathrm{rad}^{2}\right)$ are obtained at optimum powers of $-1 \mathrm{dBm}$ and $0 \mathrm{dBm}$ for LP01 and LP11, respectively.

\section{B. Phase noise versus transmission distance}

In order to explore the effect of fiber nonlinearity on the system performance, the NPN variance versus transmission distance for 2-mode MDM system is shown in Fig. 3. During obtaining results, the power of three spatial modes is set to $0 \mathrm{dBm}$. The length of each span is set to $50 \mathrm{~km}$. As expected, the impact of fiber nonlinearity on the performance of the LP11a and LP11b spatial modes is less than that for 
LP01 mode. Figs. 3(a) and 3(b) illustrate the NPN variance of both SPM and XPM effects, respectively. For all modes, the XPM has the greatest effect on the total phase noise as it increased rapidly with the increase of transmission distance (c.f. Fig. 3(c)). Because of both SPM and XPM noises are uncorrelated, the variance of total phase noise is equal to the sum of their variances.

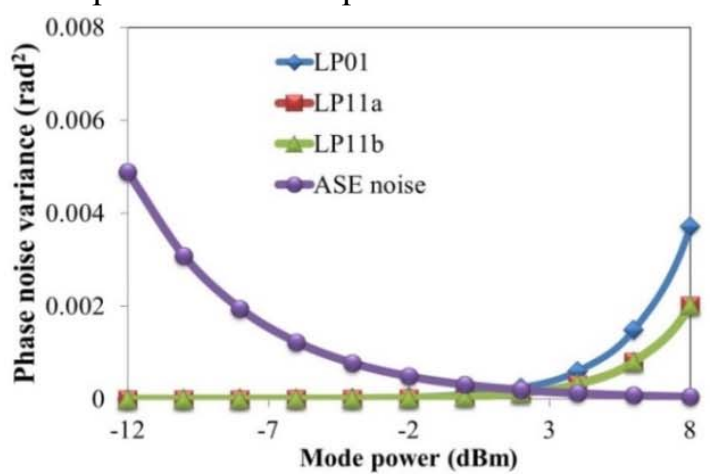

(a)

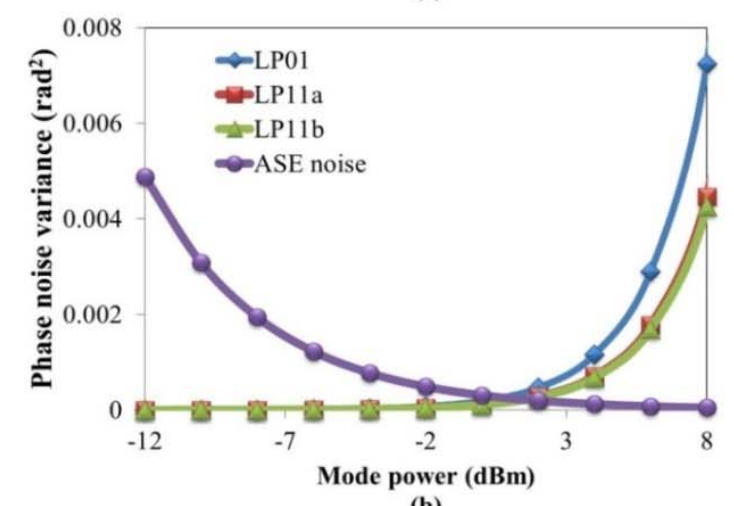

(b)

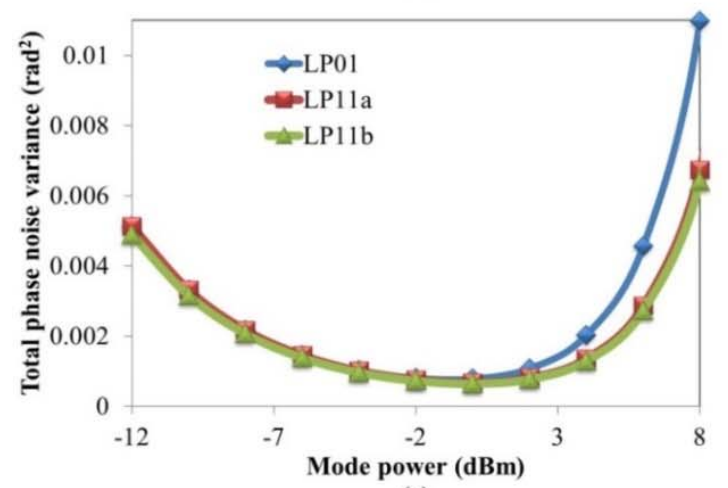

(c)

Fig.2. THE VARIANCE OF NPN AgAinst POWER FOR LP01, LP11A, AND LP11B MODES: A) SPM B) XPM C) VARIANCE OF TOTAL NPN.

\section{EVM measurements}

In Fig. 4, we demonstrate the influence of mode power on error vector magnitude (EVM) for 2-mode 4-QAM MDM system. The length of the optical link is adjusted to $250 \mathrm{~km}$ ( 5 spans). The symbol rate is fixed at $20 \mathrm{Gsymbol} / \mathrm{s}$. In contrast to the total NPN variance versus mode power (cf. Fig. 2(c)), a similar tendency can be seen in variation EVM with mode power. EVMs of all spatial modes initially decrease from 0.15 until they reach $0.072,0.0647$, and 0.0642 at the power of $-1,0$ and $0 \mathrm{dBm}$ for LP01, LP11a, and LP11b, respectively. Then, when the mode power rises beyond these values, the EVMs increase exponentially since the impact of fiber nonlinearity is dramatically boosted at higher mode powers. 


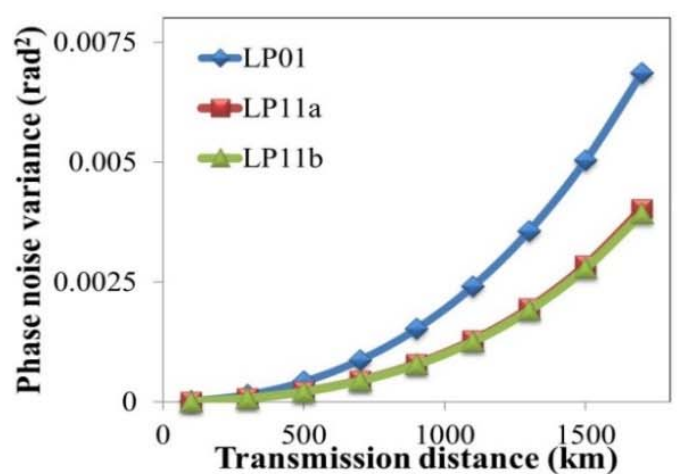

(a)

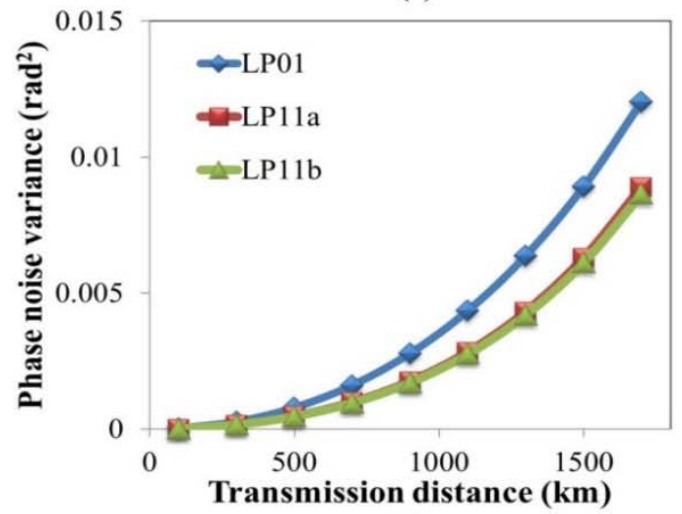

(b)

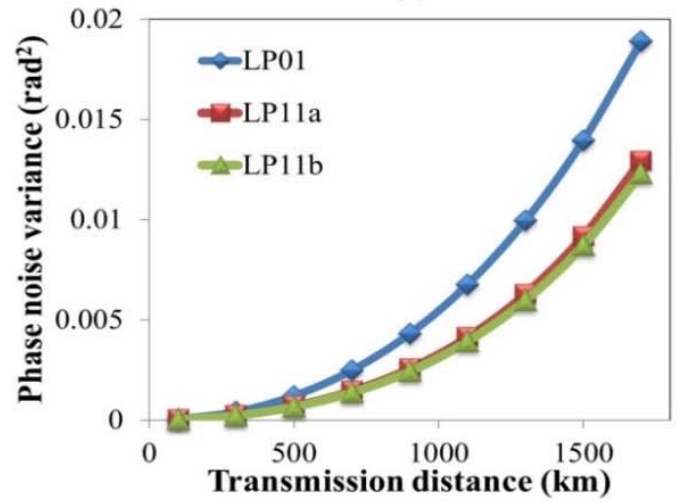

(c)

Fig. 3. THE VARIANCE OF NPN AGAINST TRANSMISSION DISTANCE:

A) SPM PHASE NOISE, B) XPM PHASE NOISE, C) TOTAL PHASE NOISE.

Finally yet important, our analytical model is valid to deal with the MDM signals that modulated with the mQAM format for any value of m-array. For example, when the signals are modulated by 4QAM format, the $u_{p}(0, t)$ is defined with $A_{p}=a_{p}+j b_{p}$, where $a_{p} \in\{-1,1\}$ and $b_{p} \in\{-1,1\}$. For higher order modulation formats $(\mathrm{m}>4)$, the $a_{p}$ and $b_{p}$ are described according to its corresponding constellation. 


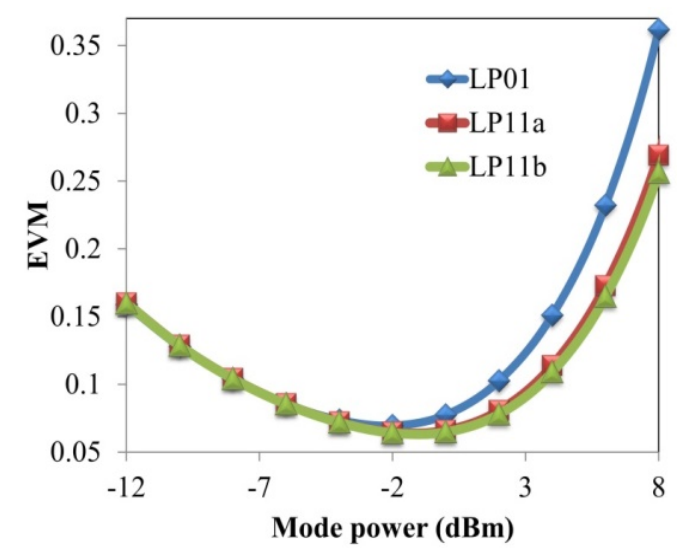

FIG.4 EVM AGAINST MODE POWER FOR LP01, LP11A, AND LP11B.

\section{CONCLUSION}

In this paper, we have developed an analytical model to describe NPN for 4-QAM MDM system using a few-mode fiber. The impact of nonlinearities and its interaction with ASE noise on system performance is analytically demonstrated. The effects of the transmission distance and mode power on NPN are investigated. The analytical results reveal that the major contribution for the nonlinear impairments is induced by XPM effect. In addition, the 4QAM signal, which is carried by LP01 mode, highly experiences fiber nonlinearity than the signals of LP11a and LP11b modes. In addition, the optimum power is occurred at $-1 \mathrm{dBm}$ and $0 \mathrm{dBm}$ for LP01 and LP11 modes, respectively.

\section{REFERENCE}

[1] J. K. Hmood, K. A. Noordin, and S. W. Harun, "Effectiveness of phase-conjugated twin waves on fiber nonlinearity in a spatially multiplexed all-optical OFDM system," Optical Fiber Technology, vol. 30, pp. 147-152, 2016.

[2] L. Xiang, G. Shen, and M. Gao, "Nonlinear propagation in multicore fiber transmission link based on coupled mode analysis," in Asia Communications and Photonics Conference, 2015, p. ASu2A. 76.

[3] R. Asif, "Advanced and flexible multi-carrier receiver architecture for high-count multi-core fiber based space division multiplexed applications," Scientific Reports, vol. 6, p. 27465, 2016.

[4] D. Richardson, J. Fini, and L. Nelson, "Space-division multiplexing in optical fibers," Nature Photonics, vol. 7, p. 354, 2013.

[5] R. Van Uden, R. A. Correa, E. A. Lopez, F. Huijskens, C. Xia, G. Li, et al., "Ultra-high-density spatial division multiplexing with a few-mode multicore fiber," Nature Photonics, vol. 8, p. 865, 2014.

[6] T. Mori, T. Sakamoto, M. Wada, T. Yamamoto, and K. Nakajima, "Few-mode fiber technology for mode division multiplexing," Optical Fiber Technology, vol. 35, pp. 37-45, 2017.

[7] J. Sakaguchi, W. Klaus, J. M. D. Mendinueta, B. J. Puttnam, R. S. Luís, Y. Awaji, et al., "Large spatial channel (36-core 3 modes) heterogeneous few-mode multicore fiber," Journal of lightwave technology, vol. 34, pp. 93-103, 2016.

[8] Y. Weng, X. He, and Z. Pan, "Space-division multiplexing optical communication using few-mode fibers," Optical Fiber Technology, vol. 36, pp. 155-180, 2017.

[9] Y. Sasaki, K. Takenaga, S. Matsuo, K. Aikawa, and K. Saitoh, "Few-mode multicore fibers for the long-haul transmission line," Optical Fiber Technology, vol. 35, pp. 19-27, 2017.

[10] P. J. Winzer and G. J. Foschini, "MIMO capacities and outage probabilities in spatially multiplexed optical transport systems," Optics Express, vol. 19, pp. 16680-16696, 2011. 
[11] K.-P. Ho and J. M. Kahn, "Linear propagation effects in mode-division multiplexing systems," Journal of lightwave technology, vol. 32, pp. 614-628, 2014.

[12] S. Mumtaz, R.-J. Essiambre, and G. P. Agrawal, "Nonlinear propagation in multimode and multicore fibers: a generalization of the Manakov equations," Journal of Lightwave Technology, vol. 31, pp. 398-406, 2013.

[13] F. Parent, J. Fade, and M. Alouini, "Orthogonality breaking through a few-mode optical fiber," Applied Optics, vol. 55, pp. 2508-2520, 2016.

[14] J. K. Hmood, K. A. Noordin, S. W. Harun, and H. M. Shalaby, "Mitigation of phase noise in alloptical OFDM systems based on minimizing interaction time between subcarriers," Optics Communications, vol. 355, pp. 313-320, 2015.

[15] J. K. Hmood and S. S. Radhi, "Influence of physical dimensions on the efficiency of phaseconjugated twin waves technique in coherent optical communication systems," Optics Communications, vol. 428, pp. 113-119, 2018.

[16] J. K. Hmood, S. W. Harun, S. D. Emami, A. Khodaei, K. A. Noordin, H. Ahmad, et al., "Performance analysis of an all-optical OFDM system in presence of non-linear phase noise," Optics Express, vol. 23, pp. 3886-3900, 2015.

[17] R.-J. Essiambre, R. W. Tkach, R. Ryf, I. Kaminow, T. Li, and A. Willner, "Fiber nonlinearity and capacity: Single-mode and multimode fibers," in Optical Fiber Telecommunications VI B, ed: Academic Press, 2013, pp. 1-37.

[18] G. P. Agrawal, "Nonlinear fiber optics," in Nonlinear Science at the Dawn of the 21st Century, ed: Springer, 2000, pp. 195-211.

[19] M. Wu and W. I. Way, "Fiber nonlinearity limitations in ultra-dense WDM systems," Journal of Lightwave Technology, vol. 22, p. 1483, 2004.

[20] A. Demir, "Nonlinear phase noise in optical-fiber-communication systems," Journal of lightwave technology, vol. 25, pp. 2002-2032, 2007.

[21] J. Hmood, K. Noordin, H. Ahmad, and S. Harun, "Performance improvement of all-optical OFDM systems based on combining RZ coding with m-array QAM," J. Optoelectron. Adv. Mater, vol. 17, pp. 33-38, 2015.

[22] A. P. T. Lau and J. M. Kahn, "Signal design and detection in presence of nonlinear phase noise," Journal of Lightwave Technology, vol. 25, pp. 3008-3016, 2007.

[23] Y. Xiao, R.-J. Essiambre, M. Desgroseilliers, A. M. Tulino, R. Ryf, S. Mumtaz, et al., "Theory of intermodal four-wave mixing with random linear mode coupling in few-mode fibers," Optics Express, vol. 22, pp. 32039-32059, 2014. 